\title{
PAIN EVALUATION IN PRETERM INFANTS USING SKIN CONDUCTANCE
} ALGESIMETER

\section{RUNNING TITLE: Do Pain Meters Work for Neonates?}

\author{
By: \\ Ahmed M, Sobithadevi DN, Mostafa S, Pore P, Maiden \\ $\mathrm{J}^{*}$, Boswell S*, Molnar M \& Reynolds TM** \\ Department of Paediatrics, Paediatric Research Nurse* and Department of Clinical Chemistry**, \\ Queen's Hospital, Burton Upon Trent, UK
}

\section{Address for Correspondence}

Dr Mansoor Ahmed Consultant

Paediatrician Queen's Hospital, Belvedere

Road

Burton-upon-Trent Staffordshire,

United Kingdom DE13 ORB

Tel: +44(0)1283 511511 Ext: 4081

Fax: +44(0)1283593031

Email: mansoor.ahmed@burtonft.nhs.uk

\subsection{ABSTRACT}

1.1 Background: Pain asessment is a challenge in patients who are unable to communicate verbally, e.g. neonates. Consequently, they may receive less

effective analgesic treatment. Visual, behavioural and physiological pain scales are used clinically but they are not always reliable, especially in premature infants. Some studies have been published on the reliability and efficacy of a Skin Conductance Algesimeter (SCA) in monitoring pain in infants. However, the data is limited by small sample size and lack of utility in specific situations such as in ventilated children and during potentially painful procedures.

1.2 Objective: To evaluate clinical usefulness of SCA as a reliable measure of pain intensity and stress response in preterm infants.

1.3 Methods: The study was conducted over a period of 3 years and parents of all infants admitted to the NNU were invited to participate in the study. The SCA was compared with simultaneous measurement of 'Premature Infant Pain Profile' (PIPP) and 'Face, Legs, Activity, Cry and Consolability (FLACC) scores during invasive / painful procedures by 2 independent assessors. Assessments were recorded for 3 minutes before, during and after the procedure. 
1.4 Results: 85 measurements were recorded. PIPP and FLACC scores started low, increased during the procedure and decreased afterwards. For all SCA measurements, there was an increase in score pre-pro and a decrease in score pro-post. However, the standard deviation for variations was wider for some measurements than for others. Paired t-test comparing Delta pre-pro with Delta pro-post for all measurements $\{$ PIPP, FLACC, Area (small) and Peaks/sec $\}$ individually showed statistically significant differences $(\mathrm{P}<0.05)$. For Area (small), there was no significant correlation between SCA raw data and PIPP/FLACC scores.

1.5 Discussion: SCA, PIPP and FLACC scores increased during the painful procedures but correlation was too imprecise to use the SCA clinically to predict or measure behavioural responses to noxious stimuli in neonates.

Keywords: Preterm, pain assessment tools, skin conductance

Abbreviations: SCA, Skin Conductance Algesimeter; NNU, Neonatal Unit; PIPP, Premature Infant Pain Profile; FLACC, Face, Legs, Activity, Cry and Consolability;

\subsection{Background}

Self-reporting is thought to be the gold standard for pain assessment. Pain assessment and hence pain management can be a challenge when patients cannot verbally communicate. This includes preterm/term neonates, infants, children with general anaesthesia and those on intensive care units. Preterm and term infants demonstrate similar or even exaggerated physiological and hormonal responses to pain compared with those observed in older children and adults (Anand KJ \& Hickey PR, 1987; Anand KJ, 1998). Infants experiencing pain during the neonatal period seem to respond differently to subsequent painful events (Grunau RE et al, 1994; Grunau RE et al, 1998). Preterm neonates are frequently exposed to multiple painful procedures after birth. Some of the painful procedures in preterm infants include heel prick blood sampling, peripheral venous blood sampling, peripheral intravenous cannulation, insertion of peripheral longlines and central lines such as umbilical arterial/venous catheterization, nasogastric/orogastric tube insertion and intubation/ventilation. Assessment of pain or stress in preterm infants admitted to the Neonatal Unit (NNU) is important but can be particularly challenging.
The inability to communicate verbally does not negate the possibility that an individual is experiencing pain. There is a substantial body of evidence to suggest that clinicians often have difficulty in assessing pain in non-verbal population. As a result, these patients often receive less effective pain treatment. Stallard et $\mathrm{al}$, in their study of everyday occurrence of pain in non-communicating children, found that while pain in these children was more common than the normal population, verbally noncommunicating children were less likely to receive active pain management (Stallard $\mathrm{P}$ et al, 2002). A number of visual, behavioural and physiological pain scales have been developed as an indirect measure of pain in tis vulnerable population. However, these are not always reliable especially in premature infants. Moreover, the mechanism for communicating pain or stressful situations through facial expressions is not well developed (Craig et al, 1993)

Severe long lasting pain and severe acute stress response may be associated with increased morbidity and mortality in a vulnerable group such as neonates (Wessel DL, 1993; Scanlon JW, 1994). Pain and stressful situations in premature infants induce an increase in heart rate, blood pressure and palmar sweating, a fall in oxygen saturation and an increase in intracranial pressure which may cause intraventricular haemorrhage 
(Pokela ML, 1994; Bellieni et al, 2003; Matthew PJ \& Matthew JL, 2003; Kopenhaver HK et al, 2008). On the other hand, an increase in heart rate and blood pressure and a fall in oxygen saturation may also follow pulmonary and cardiovascular diseases.

Even though, assessment of pain by the Skin Conductance Algesimeter (SCA) has been studied in artificially ventilated children (Gjerstad AC et al, 2008), there is a dearth of published data on its use in artificially ventilated preterm infants. According to Pokela ML, tracheal suction and routine procedures in respiratory- distressed neonates results in prolonged hypoxemia compared to neonates subjected to the same procedures after receiving pain relief (Pokela ML, 1994).

The researchers believe that the SCA detects nociceptive pain fast and continuously, specific to the individual and with higher sensitivity/specificity than other available objective methods (Storm H, 2008). However, there is paucity of data regarding its use in preterm infants which is limited by small sample size and assessment of its efficacy during the painful procedures such as heal prick blood sampling (Gladman G et al, 1990; Storm H, 2000).

The changes observed by SCA reflect the sympathetic nervous system influenced by changes in emotions, which releases the acetylcholine that acts on muscarinic receptors. Emotional sweating is activated through skin sympathetic nerves from the cerebral cortex and is not influenced by environmental temperatures (Storm $\mathrm{H}$,

2008). Each time this part of the sympathetic nervous system is activated, palmar and plantar sweat glands are filled and a spontaneous wave of skin conductance occurs (Venable PH \& Christies MJ, 1980) The number and amplitude of the waves increase with increased activity (Storm H, 2008). The skin resistance is reduced and skin conductance increases before the sweat is reabsorbed which subsequently leads to decreased skin conductance again. This creates a skin conductance peak; the size of which depends on how forcefully the skin sympathetic nerve is firing. The skin conductance peak is specific for the stimulus and is evident within 1-2 seconds after stimulation. The skin sympathetic nerve releases acetylcholine that acts on muscarine receptors and is therefore not influenced by neuromuscular blockade, adrenergic receptor active agents, or changes in blood volume (Gjerstad AC, 2008).

Landrot IDR et al, in their study analysed and compared autonomic nervous system activity indices in a group of 30 premature infants and a reference group of

14 full-term age-matched newborn infants and noted that sympathetic and even more markedly, parasympathetic activities were very low in premature infants compared to the reference full-term group (Landrot IDR et al, 2007). However, numerous published studies have concluded that foetal stress responses to invasive procedures are found from at least 23 weeks gestational age and by 24 weeks gestational age, all the neurological structures necessary for nociception are developed (Ginnakoulopoulos X, 1994; Anand KJ \& Hickey PR, 1987). Storm H in her study in preterm infants concluded that measurements of spontaneous skin conductance activity showed stress responses to heel stick from at least 29 weeks gestational age in healthy premature infants (Storm H, 2000).

Exposure to prolonged or severe pain may increase neonatal morbidity (Anand $\mathrm{KJ}$ et al, 1999). Taddio et al suggested that pain experienced early in life by term infants may exaggerate affective and behavioural responses during subsequent painful events (Taddio A et al, 1997). Neonates who are exposed to numerous painful and noxious stimuli between postconceptual weeks 28 and 32 show different behavioural and physiological responses to pain compared with neonates of a similar postconceptual age who do not have such experiences (Johnston CC

\& Stevens BJ, 1996). Surgical stress in neonates, demonstrated by increased levels of stress hormones, has also been associated with an increased incidence of postoperative complications and even deaths (Anand KJ, 
1998; Anand KJ \& Hickey PR, 1987; Anand KJ

\& Hickey PR, 1992). Other adverse consequences of insufficient pain therapy are hemodynamic instability, hypoxemia or increased

intracranial pressure especially in premature infants (Bellieni CV et al, 2003) which could potentially result in severe intraventricular haemorrhage (Anand $\mathrm{KJ}$ et al,

1999). Therefore, a more objective method of assessing pain or stressful situations which has the characteristics of being a fast-reacting, objective, sensitive, specific and continuous method to monitor pain in preterm infants is warranted in NNU

setting.

\subsection{Objective:}

To evaluate the clinical usefulness of SCA as a reliable and valid measure of pain intensity and stress response in preterm infants.

\subsection{Methods:}

\subsection{Subjects:}

All babies born at $<35$ weeks gestation and/or birth weight of $<1.8 \mathrm{~kg}$ (or $<2 \mathrm{~kg}$ if intrauterine growth retarded) are admitted to our NNU. In addition, newborn babies with various other problems including those requiring resuscitation at birth, fits, breathing/feeding difficulties etc are also admitted to the NNU. Majority (but not all) babies require one or more invasive and painful procedures like venepuncture, cannulation, nasogastric tube insertion, endotracheal suctioning, percutaneous long line insertion, insertion of central lines like umbilical arterial and umbilical venous catheterization, immunization, intra muscular or sub-cutaneous injection and heal prick blood sampling.

3.2 Inclusion criteria: Parents of all preterm infants (<37 weeks gestation) admitted to the NNU were invited to participate in the study. Only infants whose parents provided signed consent were included.
3.3 Exclusion criteria: Clinically unstable patients and patients with intraventricular haemorrhage (IVH).

\subsection{Duration and sample size:}

The study was conducted over a period of three years (January 2010 to December

2012). We recorded 85 complete measurement sets during the study period (no more than 3 measurements per patient).

Sample size calculations were carried out on the basis of 'odds of grade $\mathrm{X}$ vs grade not- $\mathrm{X}$ '. The relevant formula for this is:

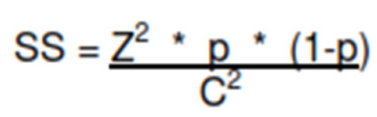

Where $\mathrm{Z}=1.96$ for $95 \% \mathrm{CI}$

$\mathrm{P}=$ proportion and $\mathrm{c}=$ confidence interval required about the proportion.

For estimating $10 \% \pm 10 \%$ of infants in grade $\mathrm{X}$, a sample size of 35 subjects and a total of 90 measurements provided a fair tolerance on proportions around $40 \%$ and very good tolerance on small proportions around $10 \%$. Our sample size was fractionally smaller than the estimated target.

\subsection{Approval:}

Approval was obtained from South Birmingham Research Ethics Committee (Ref \# 09/H1207/170; Chairman, Dr. Simon Bowman), and Research and Development

Committee at Queen's Hospital, Burton Upon Trent.

\subsection{Apparatus and software:}

The SCA (Med-Storm Innovation AS, Norway) is a device that primarily measures changes in skin conductance in real time to assess pain in the patient. Skin conductance activity was measured by alternating current at $88 \mathrm{~Hz}$. An applied voltage of $50 \mathrm{mV}$ and a three electrode system were used. The three electrode system comprised of a measuring electrode, a counter 
current electrode, and a reference voltage electrode (Figure 1).

Figure 1: System overview sketch

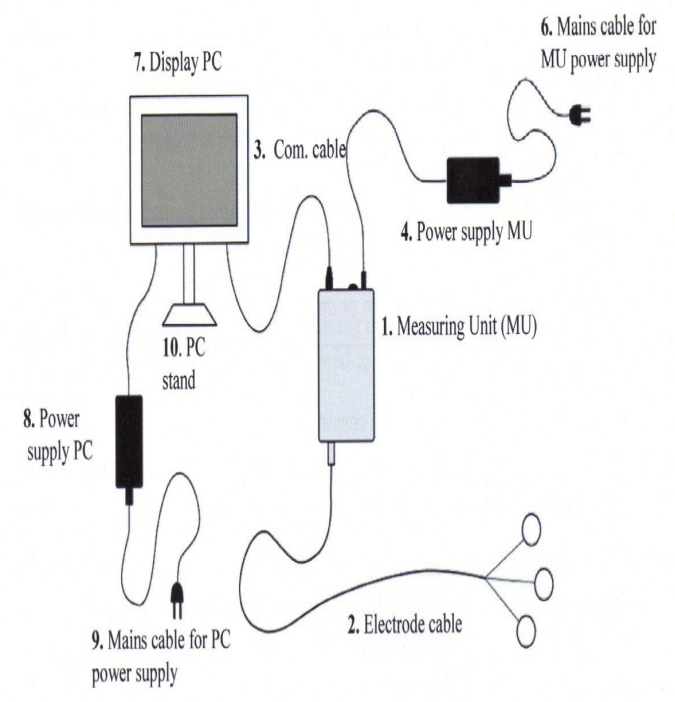

SCA electrodes were attached to the infant's right foot 10 minutes before the procedure using disks of double sided adhesive tape (Figure 2).

Figure 2: Skin electrode placement

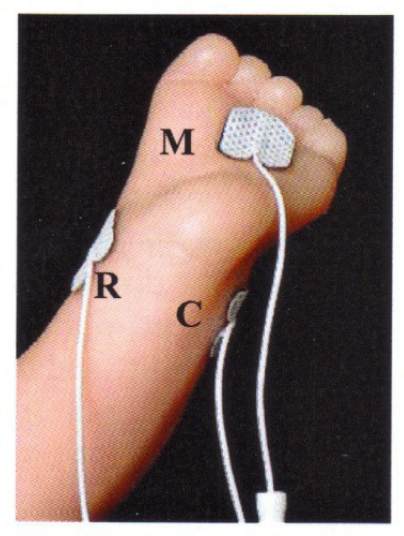

The software program recorded and counted the number of waves per second, by defining the valleys and peaks, and calculated the mean of the amplitudes of the waves and the mean baseline in the study period for the spontaneous skin conductance activity. The amplitude of the wave was calculated from the bottom of the valley before the peak to the height of the peak. The slope was defined as the mean distance of the valley to the peak/time to reach the peak.

\subsection{Procedure:}

Testing was conducted on the NNU. When a clinical decision was made to undertake an invasive or potentially painful procedure in the recruited infants, a simultaneous physiological response using Faces, Legs, Activity, Cry and Consolability (FLACC) (Merkel SI, 1997; Malviya S, 2006) and behavioural measure using Premature Infant Pain Profile (PIPP) (Stevens B et al, 1996) was recorded by two researchers independently. This was coupled with concurrent measurement of skin conductance activity by the third researcher. All of these measurements were recorded for three minutes before, during and for three minutes after the procedure.

The SCA analyser software reports 4 standard measurements:

- Area(Huge): measures the total peak area compared with a baseline set by the first peak minimum.

- Area(Small): measures the areas of each individual peak compared against a rolling baseline.

- Peaks/Sec: measures the number of peaks per second

- AvPeaks: measures average number of peaks in a time window

In addition, all data sets were examined as rolling time windows to identify the maximum number of peaks in a 15 second time window in each of the three procedure segments i.e. pre (before), pro (during) and post (after).

\subsection{Statistical analysis}

Data was analysed using Microsoft Office Excel. 
The means of the independent PIPP and FLACC measurements were calculated and the change in measurement pre-procedure to pro-procedure (Delta-prepro) and pro-procedure - postprocedure (Delta-propost) were calculated. For each of the SCA measurements, similar Delta measurements were calculated. Data was analysed to identify significance of measurement [i.e. did the variable provide any indication of pain and to identify correlation between SCA measurements and PIPP/FLACC scores.

\subsection{Results:}

4.1 PIPP \& FLACC scores: descriptive statistics: Tables $1 \mathrm{a} \& \mathrm{~b}$ demonstrate that both PIPP and FLACC scores started low, increased during the procedure and decreased afterwards. The Deltas also clearly show scores increase and then decrease as the procedure starts and ends respectively.

Table 1a: FLACC scores. Mean/SD for mean of paired scores for each stage of measurement and Delta. It can be seen that FLACC scores were low before the procedure (FLACCPro), increased during the procedure (FLACCPro) and returned to basal levels afterwards (FLACCPost). The Delta scores therefore changed accordingly with a positive change Pre-> and a negative change Pro-Post.

\begin{tabular}{|c|c|c|c|c|c|}
\hline & FLACCPre & FLACCPro & FLACCpost & $\begin{array}{l}\text { FLACC } \\
\text { Deltaprepro }\end{array}$ & \begin{tabular}{|l} 
FLACC \\
Deltapropost
\end{tabular} \\
\hline Mean & 0.78824 & 5.305882 & 0.717647 & 4.517647 & -4.58824 \\
\hline SD & 1.72409 & 3.194204 & 1.753308 & 3.414297 & 3.409699 \\
\hline Min & 0 & 0 & 0 & -3 & -10 \\
\hline Max & 9.5 & 10 & 10 & 10 & 2.5 \\
\hline
\end{tabular}

Table 1b: PIPP scores. Mean/SD for mean of paired scores for each stage of measurement and Delta. Changes of scores are similar to those shown in table 1a.

\begin{tabular}{|l|l|l|l|l|l|}
\hline & & & & & \\
& PIPPPre & PIPPPro & PIPPpost & PIPPDeltaprepro & PIPPDeltapropost \\
\hline Mean & 3.088235 & 8.294118 & 3.923529 & 5.205882 & -4.37059 \\
\hline SD & 1.404026 & 3.623086 & 1.88115 & 3.588419 & 3.750518 \\
\hline Min & 0.5 & 1 & 0 & -0.5 & -12.5 \\
\hline Max & 6.5 & 15.5 & 12 & 12.5 & 5.5 \\
\hline
\end{tabular}

4.2 SCA scores: descriptive statistics

Tables $2 \mathrm{a}$ to $2 \mathrm{e}$ demonstrate that for all measurements there was an increase in score prepro and a decrease in score pro-post. However, the standard deviation for those variations was wider for some measurements than for others.

Table 2a: Huge Peaks: Mean/SD for each stage of measurement and Delta 


\begin{tabular}{|l|l|l|l|l|l|}
\hline & & & & & \\
& Hugepre & HugePro & HugePost & HugeDeltaprepro & HugeDeltapropost \\
\hline Mean & 11.43659 & 50.10741 & 17.33 & 38.67082 & -32.7774 \\
\hline SD & 33.69738 & 215.6441 & 86.98868 & 217.5662 & 156.1661 \\
\hline Min & 0 & 0 & 0 & -198.79 & -1284.34 \\
\hline Max & 224.89 & 1432.28 & 787.57 & 1423.71 & 71.29 \\
\hline
\end{tabular}

Table 2b: Small Peaks: Mean/SD for each stage of measurement and Delta

\begin{tabular}{|l|l|l|l|l|l|}
\hline & & & & & \\
& Smallpre & Smallpro & Smallpost & SmallDeltaprepro & SmallDeltapropost \\
\hline Mean & 2.551647 & 3.989882 & 2.251765 & 1.438235 & -1.73812 \\
\hline SD & 3.844117 & 7.314786 & 5.380378 & 5.245798 & 6.045893 \\
\hline Min & 0 & 0 & 0 & -11.48 & -36.94 \\
\hline Max & 21.17 & 39.68 & 31.79 & 28.41 & 19.25 \\
\hline
\end{tabular}

Table 2c: Peaks per second: Mean/SD for each stage of measurement and Delta

\begin{tabular}{|l|l|l|l|l|l|}
\hline & & & & & \\
& PersecPre & PersecPro & persecPost & PersecDeltaprepro & PersecDeltapropost \\
\hline Mean & 0.061882 & 0.079529 & 0.040471 & 0.017647 & -0.039058824 \\
\hline SD & 0.080836 & 0.067293 & 0.068172 & 0.066362 & 0.069857398 \\
\hline Min & 0 & 0 & 0 & -0.18 & -0.23 \\
\hline Max & 0.51 & 0.36 & 0.35 & 0.19 & 0.21 \\
\hline
\end{tabular}

Table 2d: Average Peaks: Mean/SD for each stage of measurement and Delta

\begin{tabular}{|l|l|l|l|l|l|}
\hline & & & & & \\
& AvPre & AvPro & AvPost & AvDeltaprepro & AvDeltapropost \\
\hline Mean & 0.097059 & 0.109647 & 0.083765 & 0.012588 & -0.02588 \\
\hline SD & 0.098426 & 0.121611 & 0.109338 & 0.118775 & 0.111828 \\
\hline Min & 0 & 0 & 0 & -0.57 & -0.37 \\
\hline Max & 0.63 & 0.87 & 0.55 & 0.41 & 0.55 \\
\hline
\end{tabular}

Table 2e: Max Peaks per process stage: Mean/SD for each stage of measurement and Delta

\begin{tabular}{|l|l|l|l|l|l|}
\hline & & & & & \\
& MaxPre & Maxpro & Maxpost & MaxDeltaprepro & MaxDeltapropost \\
\hline Mean & 0.236471 & 0.307765 & 0.247647 & 0.071294 & -0.06012 \\
\hline SD & 0.202342 & 0.203677 & 0.767619 & 0.198865 & 0.769526 \\
\hline $\min$ & 0 & 0.01 & 0 & -0.66 & -0.6 \\
\hline $\max$ & 1.13 & 1.33 & 7 & 0.73 & 6.73 \\
\hline
\end{tabular}

\subsection{Which data is discriminatory?}

To evaluate whether the changes in scores was statistically significant, the Delta values for all measurement sets were compared by paired t-test. Table 3 shows that PIPP, FLACC, Area (small) and Peaks/sec all showed statistically significant differences $(\mathrm{P}<0.05)$. Other parameters were not significant and were not analysed further. 
Table 3: Paired t-test comparing Delta-prepro with Delta-propost for all measurements.

N.S. = Not Significant

Only PIPP, FLACC, Area(small) and Peaks/sec were statistically significant.

\begin{tabular}{|l|l|l|l|l|l|l|l|}
\hline & FLACC & PIPP & Area(Huge) & Area(small) & Peaks/sec & AvPeaks & MaxPeaks \\
\hline mean & 9.105882 & 9.576470588 & 71.44824 & 3.176353 & 0.056706 & 0.038471 & 0.131412 \\
\hline SD & 6.404098 & 7.001915504 & 368.0506 & 10.24003 & 0.120034 & 0.185206 & 0.788457 \\
\hline SEM & 0.694622 & 0.759464368 & 39.92069 & 1.110687 & 0.013019 & 0.020088 & 0.08552 \\
\hline t & 13.10912 & 12.60950611 & 1.789755 & 2.859808 & 4.355467 & 1.915065 & 1.536617 \\
\hline P & $5.09 \mathrm{E}-22$ & $4.49031 \mathrm{E}-21$ & $\begin{array}{l}0.077097 \\
\text { N.S. }\end{array}$ & 0.005346 & $3.73 \mathrm{E}-05$ & $\begin{array}{l}0.058887 \\
\text { N.S. }\end{array}$ & $\begin{array}{l}0.128146 \\
\text { N.S. }\end{array}$ \\
\hline
\end{tabular}

4.4 Correlation between PIPP/FLACC and SCA data

Table 4 shows that for Area (small), there was no significant correlation with PIPP/FLACC scores. On the other hand, for Peaks / sec there was a statistically significant correlation with PIPP/FLACC scores. This would appear to suggest that Peaks./ sec might be a useful marker.

Table 4: Correlation between PIPP and FLACC scores and SCA scores.

Correlation is between similar data sets e.g. DeltaFLACCPrepro and

DeltaArea(small)prepro.

In all cases, $\mathrm{N}=85$

It is clear that only data for Peak/sec is statistically significant.

\begin{tabular}{|l|l|l|}
\hline & FLACC & PIPP \\
\hline Pre $\rightarrow$ Pro & & \\
\hline Area(Small) & $\mathrm{r}=0.1320$ & $\mathrm{r}=0.1188$ \\
& $\mathrm{t}=1.2057$ & $\mathrm{t}=1.0832$ \\
& $\mathrm{P}=0.2314$ & $\mathrm{P}=0.2818$ \\
\hline Peaks/sec & $\mathrm{r}=0.5055$ & $\mathrm{r}=0.4536$ \\
& $\mathrm{t}=5.3046$ & $\mathrm{t}=4.6083$ \\
& $\mathrm{P}=9.2 \mathrm{E}-7$ & $\mathrm{P}=1.45 \mathrm{E}-5$ \\
\hline Pro $\rightarrow$ Post & $\mathrm{r}=-0.0210$ & $\mathrm{r}=-0.1000$ \\
\hline Area(Small) & $\mathrm{t}=0.1900$ & $\mathrm{t}=0.9104$ \\
& $\mathrm{P}=0.8500$ & $\mathrm{P}=0.3652$ \\
\hline Peaks/sec & $\mathrm{r}=0.4412$ & $\mathrm{r}=0.4004$ \\
& $\mathrm{t}=4.4525$ & $\mathrm{t}=3.9566$ \\
& $\mathrm{P}=2.62 E-5$ & $\mathrm{P}=0.00016$ \\
\hline
\end{tabular}

Figure 3 shows the relationship between FLACC and Peaks/sec, and Figure 4 shows the relationship between PIPP and Peaks/sec. It is clear from the graphs that although there is correlation between the variables, the change in peaks/sec is widely variable compared to the change in PIPP or FLACC, such that an average change in peaks/sec could correspond to any change in PIPP or FLACC. Thus although the data is mathematically correlated, at a clinical level the correlation is too imprecise to use the SCA to predict or measure behavioural responses to noxious stimuli in neonates. 
Figure 3: Plots of Delta FLACC vs Delta Peaks/sec.
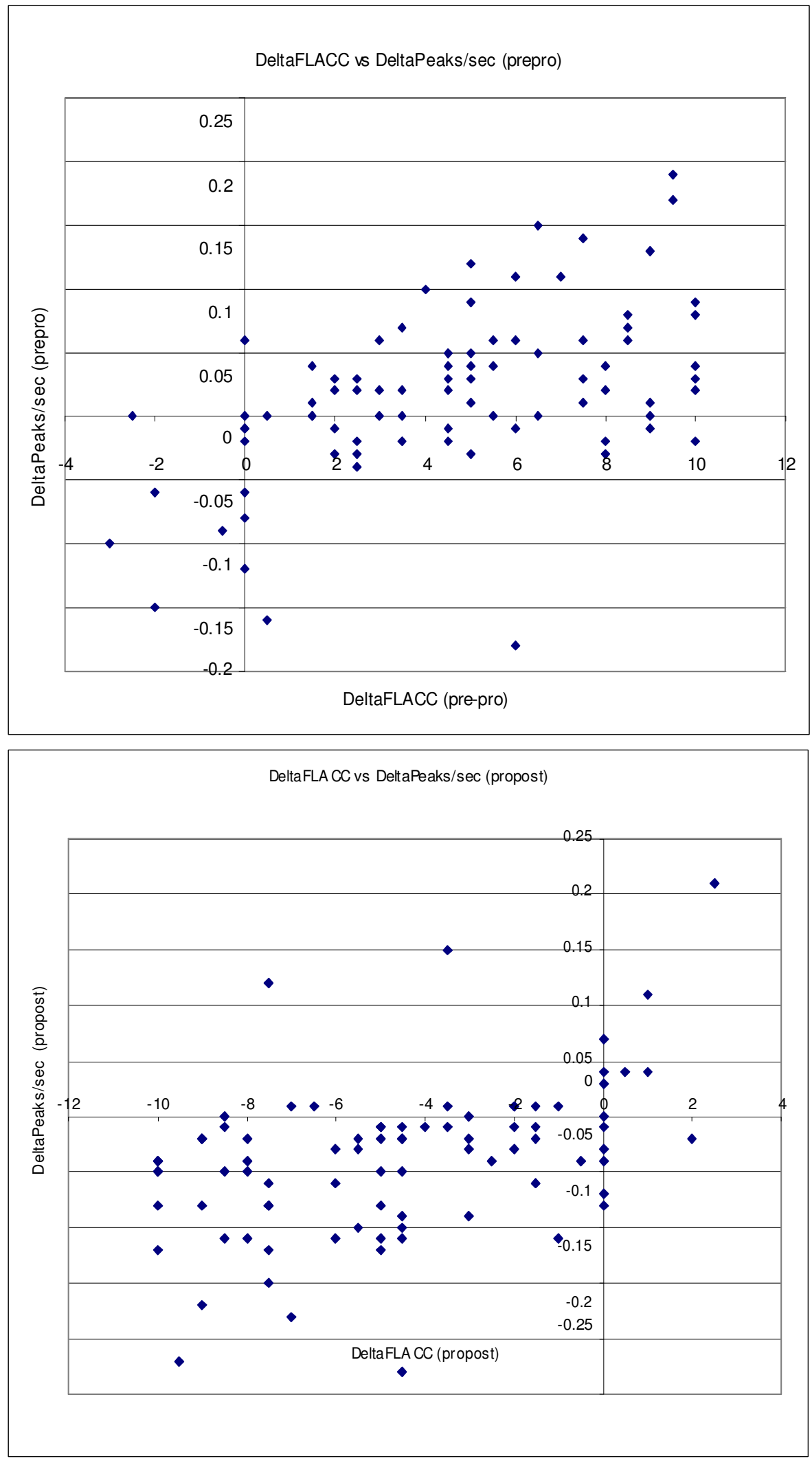
Figure 4: Plots of Delta PIPP vs Delta Peaks/sec

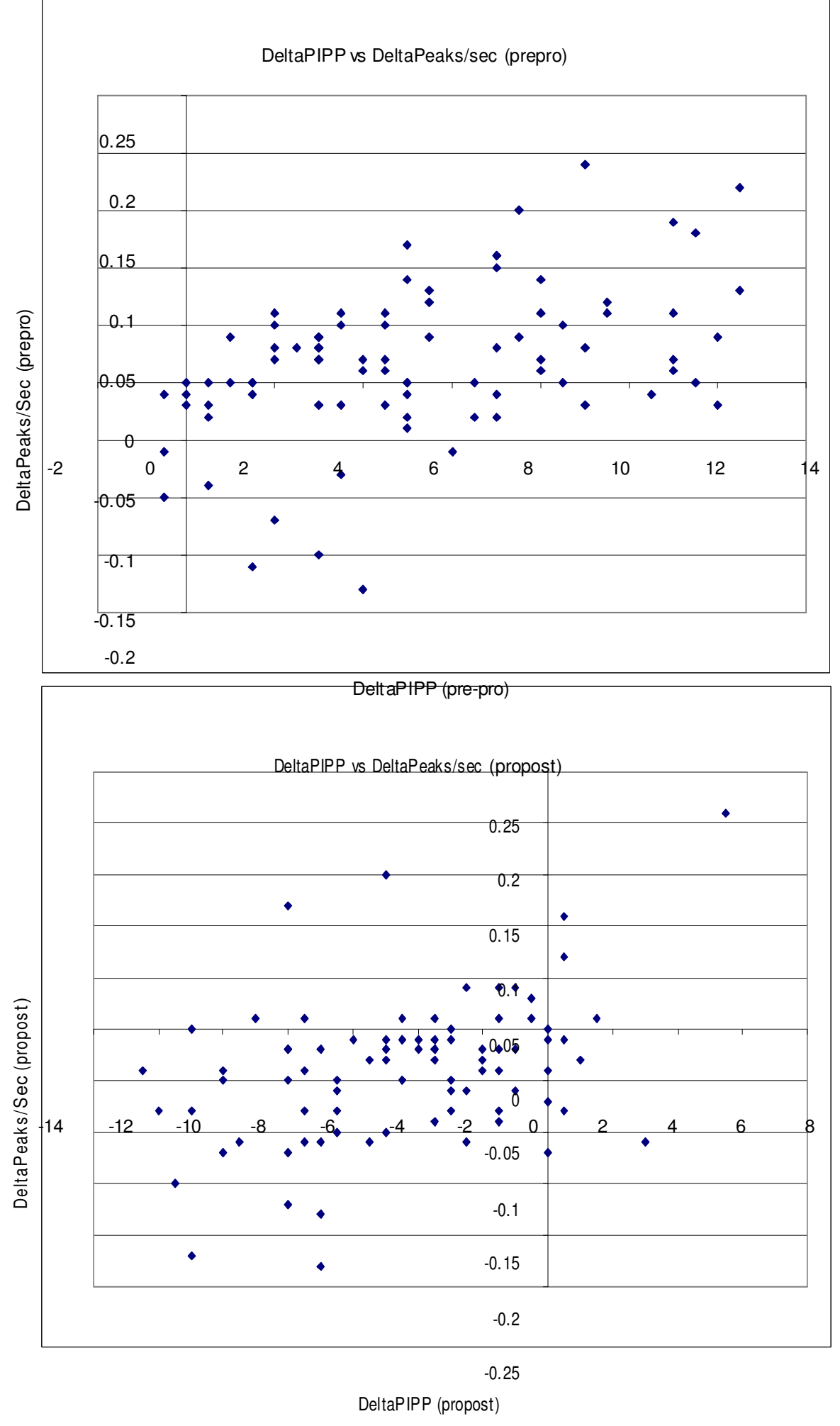




\subsection{Discussion:}

A position statement by the American Academy of Pediatrics and the Canadian Paediatric Society recommends that in order to evaluate and reduce the stress and pain experienced by neonates, validated measures and assessment tools must be used consistently and the assessments should continue as long as the neonate requires treatment for stress or pain. They also endorse that health care professionals should use appropriate environmental, nonpharmacological (behavioural), and pharmacological interventions to prevent, reduce, or eliminate the stress and pain of neonates (Committee on Fetus and Newborn, 2000).

To the best of our knowledge, the current study is the first to describe the use of SCA as a tool for pain assessment in preterm infants during painful procedures. Unlike Gladman's study where no babies less than 36 weeks had a change in their skin conductance after the heel prick, we observed that SCA, PIPP as well as FLACC scores increased during the painful procedures (Gladman, $G$ \& Chiswick ML, 1990). Of the five possible data outputs from the SCA software, only two showed a significant procedure-related change but only one of these (Peaks/sec) showed a statistically significant correlation with PIPP/FLACC. However, despite this correlation there was insufficient discriminatory power for Peaks/sec to be a useful clinical marker.

Adequate pain control is crucial to the wellbeing of preterm neonates on the NNU. There is a growing concern that even constant exposure to ambient noise in NNU that often exceeds recommended levels puts preterm infants at high risk for adverse health effects (Elisha M, et al, 2011). In their study, Anand and Hickey established decreased incidence of sepsis, metabolic acidosis, and disseminated intravascular coagulation with fewer postoperative deaths among critically ill neonates who received deep anaesthesia and postoperative analgesia with higher doses of opioids (Anand KJ \& Hickey PR, 1992). On the other hand, tolerance, physical dependence, and withdrawal abstinence can potentially ensue after prolonged administration of drugs used for sedation and analgesia in paediatric intensive care population (Tobias JD, 2000). Hence, it is vital to find the right balance between suitable pain control without discomfort and excessive use of sedative and analgesic drugs.

One of the limitations of SCA is the possible movement artefacts when using this kind of equipment in awake babies. The other limitation of SCA when assessing pain is that SCA reflects palmar sweat gland filling and hence ultimately sympathetic tone. Therefore, all the factors that influence sympathetic tone may, in theory, alter the data captured by SCA henceforth its parameters to painful stimuli. Moreover, there is currently no reliable, validated and standardised tool for the assessment of acute pain in preterm infants on NNU. PIPP and FLACC used in our study and various other scoring systems used in previous trials are highly

observer-dependent. Therefore, all the scoring systems/tool developed so far lack clearly specified sensitivities/specificities in the description of pain states as the subjectivity of pain does not allow comparison with absolute values for pain. This becomes even more complicated in the context of preterm infants on the NNU.

In their study, Strehle E-M and Gray found a weak correlation between self- reported pain scores and the rate of fluctuations in skin conductance for females but not for males and SCA was not found to be of significant benefit to patients in 
minor injuries unit (Strehle E-M \& Gray WK, 2013). Even though Hullett et al determined that SCA accurately predicted the absence of moderate to severe pain in postoperative paediatric patients, the authors question the applicability of this methodology in the clinical setting (Hullett B et al, 2009).

We conclude that the correlation between SCA, PIPP and FLACC scores is too imprecise to use SCA at a clinical level to predict or measure behavioural responses to noxious stimuli in neonates and the clinical applicability of this method in NNU setting remains to be seen. Even if SCA or other neonatal pain assessment scores are used in clinical setting, it is important that the caregivers should use their clinical judgement to find out whether the warning is caused by pain or another stressor. Further research is therefore required to define the precise role of SCA assessments in the management of neonatal pain in NNUs. Further improvements in the SCA software technology with immediate availability of pain score data on SCA monitor at the point of care in real time may enable neonatologists to accept this as a validated pain assessment tool. Once developed and universally accepted, an ideal pain assessment tool is likely to produce real time measurements, accurately predict pain with fast reaction time and generate an index valid for preterm as well as term infants. Furthermore, simple, light weight, user friendly and low cost equipment would attract its widespread use in the paediatric and neonatal clinical settings.

\section{Acknowledgement: Funding:}

Funding was received from the local Research \& Development Committee for the purchase of SCA.

\section{Competing interests:}

None.

\section{Previous Presentations}

A poster presentation of this study has been accepted for the European Academy of Paediatric Societies (EAPS) meeting in Barcelona, Oct 2014. 


\subsection{References}

Anand KJ. Clinical importance of pain and stress in preterm neonates.

Biol Neonate. 1998;73:1-9

Anand KJ, Hickey PR. Pain and its effects in the human neonate and fetus. N Engl J Med. 1987;317:1321-1329

Anand KJ, Hickey PR. Halothanemorphine compared with high-dose sufentanil for anesthesia and postoperative analgesia in neonatal cardiac surgery. $N$ Engl J Med. 1992;326(1):1-9

Anand KJ, McIntosh N, Lagercrantz H, Pelausa E, Young TE, Vasa R.

Analgesia and sedation in preterm neonates who require ventilator support: results from the Neonatal Outcome and Prolonged Analgesia in Neonates trial. Arch Pediatr Adolesc Med: 1999; 153:331-338

Bellieni CV, Burroni A, Perrone S, et al. Intracranial pressure during procedural pain. Biol Neonate 2003;84:202-205.

Committee on Fetus and Newborn, Committee on Drugs, Section on Anesthesiology, Section on Surgery and Canadian Paediatric Society, Fetus and Newborn Committee. Prevention and Management of Pain and Stress in the Neonate. Pediatrics 2000;105;454-461

Craig KD, Whitfield MF, Grunau RVE, Linton J, Hadjistavropoulos HD. Pain in preterm neonate: behavioural and physiological indices. Pain 1993;52:287-299

Elisha M Wachman EM, Amir Lahav A. The effects of noise on preterm infants in the NICU. Arch Dis Child Fetal Neonatal Ed 2011;96:F305F309

Ginnakoulopoulos X, Sepulveda W, Kourtis P, Glover V, Fisk NM. Fetal plasma cortisol and beta-endorphin response to intrauterine needling.
Lancet 1994;344:77-81.

Gjerstad AC, Wagner K, Henrichsen T and Storm H. Skin Conductance Versus the Modified COMFORT Sedation Score as a Measure of Discomfort in Artificially Ventilated Children. Pediatrics 2008;122:e848-e853

Gladman G, Chiswick ML. Skin conductance and arousal in the newborn. Arch Dis

Child 1990;65:1063-1066

Grunau RVE, Whitfield MF, Petrie JH. Pain sensitivity and temperament in extremely low-birth-weight premature toddlers and preterm and full-term controls. Pain. 1994;58:341346

Grunau RE, Whitfield MF, Petrie J. Children's judgements about pain at age 8-10 years: do extremely low birthweight $(\leq 1000 \mathrm{~g})$ children differ from full birthweight peers? J Child Psychol Psychiatry. 1998;39:587-594

Hullett B, Chambers N, Preuss,J, Zamudio I, Lange J, Pascoe E and Ledowski T. Monitoring Electrical Skin Conductance-A Tool for the Assessment of Postoperative Pain in Children? Anesthesiology 2009; 111:513-7

Johnston CC, Stevens BJ. Experience in a neonatal intensive care unit affects pain response. Pediatrics. 1996;98:925-930

Kopenhaver Haidet K, Adkin C, Rebstock S, et al. Measures of stress vulnerability in LBW infants: an integrative biobehavioral approach to stress reactivity measurement, Abstract Gravens conference, Florida 2008.

Landrot IDR, Roche F, Pichot V et al. Autonomic nervous system activity in premature and full-term infants from 
theoretical term to 7 years. Auton Neurosci.

2007;136:105-9.

Malviya S, Voepel-Lewis, T. The revised FLACC observational pain tool: improved reliability and validity for pain assessment in children with cognitive impairment. Paediatric Anaesthesia 2006;16[3]:258-265.

Mathew PJ, Mathew JL. Assessment and management of pain in infants. Postgrad Med J 2003;79:438-443.

Merkel SI, Voepel-Lewis T, Shayevitz JR and Malviya S. The FLACC: a behavioral scale for scoring postoperative pain in young children. Pediatr Nurs

1997;23(3):293-7

Pokela ML. Pain relief can reduce hypoxemia in distressed neonates during routine treatment procedures. Pediatrics 1994;93:379-383.

Scanlon JW. Appreciating neonatal pain. Physiological responses of premature infants to a painful stimulus. Nurs Res 1994;43:226-231

Stallard P, Williams L, Velleman R, Lenton S, McGrath PJ. Intervening factors in caregivers' assessments of pain in non-communicating children. Developmental Medicine \& Child Neurology. 2002;44:212-214

Stevens B Johnston C et al. Premature Infant Pain Profile: Development and initial validation. Clin J Pain 1996;12:13-22
Storm H. Skin conductance and the stress response from heel stick in preterm infants. Arch Dis Child Fetal Neonatal Ed 2000;83:F143-F147

Storm $H$. Changes in skin conductance as a tool to monitor nociceptive stimulation and pain. Current Opinion in Anaesthesiology 2008;21:796-804

Strehle E-M and Gray WK. Comparison of skin conductance measurements and subjective pain scores in children with minor injuries. Acta Pediatrica 2013;102:e502-506

Taddio A, Katz J, llersich AL, Koren G. Effect of neonatal circumcision on pain response during subsequent routine vaccination. Lancet. 1997;349:599-603

Tobias JD. Tolerance, withdrawal, and physical dependency after long-term sedation and analgesia of children in the pediatric intensive care unit. Crit Care Med. 2000;28(6):2122-2132

Venables $\mathrm{PH}$, Christies MJ. Electrodermal activity. In: Martin I, Venables $\mathrm{PH}$, eds.

Techniques in psychophysiology. Chichester: John Wiley \& Sons Ltd, 1980;3-67.

Wessel DL. Hemodynamic responses to perioperative pain and stress in infants.

Crit Care Med 1993;21:361-362. 